INJE-TP-05-04

hep-th/0506031

\title{
Unitarity issue in BTZ black holes
}

\author{
Y. S. Myung ${ }^{1,2 *}$ and H. W. Lee ${ }^{1 \dagger}$ \\ ${ }^{1}$ Institute of Mathematical Science and School of Computer Aided School \\ Inje University, Gimhae 621-749, Korea \\ ${ }^{2}$ Institute of Theoretical Science, University of Oregon, Eugene, OR 97403-5203, USA
}

\begin{abstract}
We study the wave equation for a massive scalar in three-dimensional AdS-black hole spacetimes to understand the unitarity issues in a semiclassical way. Here we introduce four interesting spacetimes: the non-rotating BTZ black hole (NBTZ), pure AdS spacetime (PADS), massless BTZ black hole (MBTZ), and extremal BTZ black hole (EBTZ). Our method is based on the potential analysis and solving the wave equation to find the condition for the frequency $\omega$ exactly. In the NBTZ case, one finds the quasinormal (complex and discrete) modes which signals for a nonunitary evolution. Real and discrete modes are found for the PADS case, which means that it is unitary obviously. On the other hand, we find real and continuous modes for the two extremal black holes of MBTZ and EBTZ. It suggests that these could be candidates for the unitary system.
\end{abstract}

\footnotetext{
*e-mail address: ysmyung@physics.inje.ac.kr
}

${ }^{\dagger}$ Email-address : hwlee@inje.ac.kr 


\section{Introduction}

Hawking's semiclassical analysis for the black hole radiation suggests that most of information in initial states is shield behind the event horizon and is never back to the asymptotic region far from the evaporating black hole[1]. This means that the unitarity is violated by an evaporating black hole. However, this conclusion has been debated for decades 2, 3, 4. The information loss paradox is closely related to the question of whether the formation and subsequent evaporation of a black hole is unitary. One of the most urgent problems in the black hole physics is to resolve the unitarity issue.

Recently, Maldacena proposed that the unitarity can be restored if one takes into account the topological diversity of gravitational instantons with the same AdS boundary in (1+2)-dimensional gravity[5]. Actually, $(1+2)$-dimensional gravity [6] is not directly related to the information loss problem because there is no physical degrees of freedom [7]. If this gravity could be considered to be part of string theory, the AdS/CFT correspondence [8, 9, 10] requires that the black hole formation and evaporating process be unitary because its boundary can be described by a unitary CFT. On later, Hawking has withdrawn his argument on the information loss and suggested that the unitarity can be restored by extending Maldacena's proposal to $(1+3)$-dimensional gravity system [11. The topological diversity is credited with the restoration of the S-matrix unitarity in the formation and evaporation of a black hole. In this approach of the Euclidean path integral, the pure AdS space plays an important role in restoring unitarity. However, the proposal which is to resolve the information loss paradox by summing over bulk topologies seems to be failed even in the (1+2)-dimensional model[12, 13].

Since the $(1+2)$-dimensional gravity and its boundary CFT can provide a prototype to compute quasinormal modes and boundary correlators exactly[14], they play a crucial role in investigating the unitarity issue. Solodukhin has tried to find an alternative view to resolving the unitarity problem by introducing a non-classical deformation of the BTZ black hole which resembles the geometry of wormhole [15]. As a result of disappearing the event horizon, real and discrete modes are found 16, 13, which means that the considering system turns out to be unitary.

In this work we focus on the study of the $(1+2)$-dimensional wave equation for a scalar field which include a massless scalar[17] as well as a dilaton derived from string theory [18, 19, 20, 21, 22]. The pure AdS spacetime provides a unitary evolution, but it is topologically trivial. It is important to find a topologically non-trivial spacetime which provides a unitary evolution. This work may put a further step to understand the unitarity in a semiclassical way because we propose the two extremal BTZ black holes 
(MBTZ, EBTZ) for the unitarity systems. Our method consists of two steps: the potential analysis using the Schrödinger-equation and obtaining its eigenvalue $E=\omega^{2}$ by solving the wave equation exactly. Actually, we translate the black hole-unitary problem into the boundary-value problem in the Schrödinger-like equation. If the Schrödinger operator $\mathcal{L}=-d^{2} / d r_{*}^{2}+V\left(r_{*}\right)$ is self-adjoint $\left(\mathcal{L}^{\dagger}=\mathcal{L}\right)$, its eigenvalue is real upon imposing appropriate boundary conditions [23, 24]. In this case there is no information loss and the unitarity is preserved. If one finds quasinormal modes, the information is lost during evolution and thus the system is not unitary ${ }^{\ddagger}$. In asymptotically AdS spacetime 25], quasinormal modes are defined as the solutions which are purely ingoing wave at the event horizon and those which vanish at infinity because the potential is growing at infinity. The last condition means that any leakage of the energy (information) is not allowed through the boundary at infinity. More precisely, we use the flux boundary condition: the ingoing flux at the horizon and the vanishing flux at infinity [26].

In a $(1+2)$-dimensional spacetime, the Einstein equation with a negative cosmological constant $\Lambda=-1 / \ell^{2}$ and $8 G_{3}=1$ provides the BTZ solution:

$$
d s_{B T Z}^{2}=-\left(-M+\frac{r^{2}}{l^{2}}+\frac{J^{2}}{4 r^{2}}\right) d t^{2}+\left(-M+\frac{r^{2}}{l^{2}}+\frac{J^{2}}{4 r^{2}}\right)^{-1} d r^{2}+r^{2}\left(d \phi-\frac{J}{2 r^{2}} d t\right)^{2}
$$

where $M$ and $J$ turn out to be the mass and angular momentum, respectively $[6]$. The above metric allows the two horizons of $r_{ \pm}^{2}=M l^{2}(1 \pm \Delta) / 2$ with $\Delta=\left(1-J^{2} / M^{2} l^{2}\right)^{1 / 2}$. Here the conditions of $|J| \leq M l$ and $M \geq 0$ are required to have the black hole spacetime. Its thermodynamic quantities of energy, Hawking temperature, Bekenstein-Hawking entropy, and heat capacity are given by $E=M, T_{H}=M \Delta / 2 \pi r_{+}, \quad S_{B H}=4 \pi r_{+}, \quad C_{J}=$ $4 \pi r_{+} \Delta /(2-\Delta)[27,28,29$. We note that the heat capacity of BTZ black hole is always positive. Hence the BTZ black hole can be thermally in equilibrium with any size of heat reservoir. This explains thermodynamically why the BTZ black hole belongs to an eternal black hole [5, 26]. In this work we consider four interesting cases. i) The non-rotating BTZ black hole (NBTZ) with $J=0: r_{+}^{2}=l^{2} M, T_{H}=\frac{r_{+}}{2 \pi l^{2}}, C_{J}=4 \pi r_{+}=S_{B H}$. ii) The pure AdS spacetime (PADS) with $M=-1, J=0: T_{H}=C_{J}=S_{B H}=0$. This case corresponds to the spacetime picture of the NS-NS vacuum state [30]. iii) The massless

\footnotetext{
${ }^{\ddagger}$ We just study the wave propagation to test how an object (the black hole) responds to an external perturbation. In the case of black hole physics, it is impossible to investigate the interior region of the event horizon using the Klein-Gordon equation for a scalar. Hence the radial part of the Klein-Gordon equation leads to the Schrödinger-like equation but not the exact Schrödinger equation because the KleinGordon equation belongs to the relativistic wave equation. The use of quantum mechanical terminology is here an analogy to understand the external perturbation intuitively. The system under consideration is not an exact quantum system. Therefore we don't need to do a further work, the self-adjoint extension of the Schrödinger operator, even if the quasinormal mode is found.
} 
BTZ black hole (MBTZ) with $M=J=0: T_{H}=C_{J}=S_{B H}=0$. This is called the spacetime picture of the R-R vacuum state. iv) The extremal BTZ black hole (EBTZ) with $|J|=l M: r_{+}^{2}=r_{-}^{2}=l^{2} M / 2, T_{H}=C_{J}=0, S_{B H}=4 \pi r_{+}$.

\section{NBTZ VS PADS}

We start with the wave propagation for a massive scalar field with mass $m$

$$
\left(\nabla^{2}-m^{2}\right) \Phi=0
$$

in the background of the non-rotating BTZ black hole. Its line element is given by $d s_{N B T Z}^{2}=-\left(-M+r^{2} / l^{2}\right) d t^{2}+\left(-M+r^{2} / l^{2}\right)^{-1} d r^{2}+r^{2} d \phi^{2}[31$. On the other hand, the pure AdS spacetime is defined by $d s_{P A D S}^{2}=-\left(1+r^{2} / l^{2}\right) d t^{2}+\left(1+r^{2} / l^{2}\right)^{-1} d r^{2}+r^{2} d \phi^{2}$. Here we set $M=l=1$ for simplicity, unless otherwise stated. Assuming a mode solution ${ }^{\S}$

$$
\Phi(r, t, \phi)=f(r) e^{-i \omega t} e^{i \ell \phi}, \ell \in \mathbf{Z}
$$

we find the radial equation

$$
\left(r^{2} \mp 1\right) f^{\prime \prime}(r)+\left(3 r \mp \frac{1}{r}\right) f^{\prime}(r)+\left[\frac{\omega^{2}}{r^{2} \mp 1}-\frac{\ell^{2}}{r^{2}}-m^{2}\right] f(r)=0,
$$

where the prime (') denotes the derivative with respect to its argument. The upper (lower) signs denote the NBTZ (PADS) cases. Introducing $f(r)=\tilde{f}(r) / \sqrt{r}$, the above equation reduces to

$$
\left(r^{2} \mp 1\right) \tilde{f}^{\prime \prime}(r)+2 r \tilde{f}^{\prime}(r)+\left[\frac{\omega^{2}}{r^{2} \mp 1}-\frac{3}{4} \mp \frac{1}{4 r^{2}}-\frac{\ell^{2}}{r^{2}}-m^{2}\right] \tilde{f}(r)=0
$$

which is suitable for the potential analysis.

First of all, it is important to see how a scalar wave propagates in the exterior of the NBTZ. For this purpose, we introduce a tortoise coordinate $2 r_{*}=\ln [(r-1) /(r+1)](r=$ $\left.-\operatorname{coth}\left[r_{*}\right]\right)$ [24, 26]. We have $r_{*} \rightarrow-\infty\left(r \rightarrow r_{+}\right)$and $r_{*} \rightarrow 0(r \rightarrow \infty)$. We transform Eq.(15) into the Schrödinger-like equation with the Schrödinger operator $\mathcal{L}_{N B T Z}$ and energy $E=\omega^{2}$

$$
-\frac{d^{2}}{d r_{*}^{2}} \tilde{f}+V_{N B T Z}\left(r_{*}\right) \tilde{f} \equiv \mathcal{L}_{N B T Z} \tilde{f}=E \tilde{f}
$$

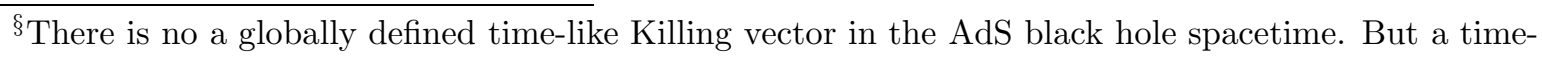
like Killing vector of $\partial / \partial t$ is future directed at the region I, the Fig. 3 in Ref. [6]. This means that an appropriate time evolution is allowed if $\mathcal{L}_{\partial t} \Phi=-i \omega \Phi$ with $\omega>0$ in this work 32 . 
where the NBTZ potential is given by 31$]$

$$
V_{N B T Z}\left(r_{*}\right)=\left[\left(\frac{3}{4}+m^{2}\right) \operatorname{coth}^{2}\left[r_{*}\right]-m^{2}+\ell^{2}-\frac{1}{2}-\left(\ell^{2}+\frac{1}{4}\right) \tanh ^{2}\left[r_{*}\right]\right] .
$$

We observe that the potential decreases exponentially to zero $\left(V_{N B T Z} \sim e^{-2 r_{*}}\right)$ as one approaches the event horizon $\left(r_{*} \rightarrow-\infty\right)$, while it goes infinity $\left(V_{N B T Z} \sim 1 / r_{*}^{2}\right)$ as one approaches infinity $\left(r_{*} \rightarrow 0\right)$. $V_{N B T Z}\left(r_{*}\right)$ looks like the right-half of $\cup$. A plane wave appears near the event horizon, whereas a genuine travelling wave does not appear at infinity. In order to obtain the solution which is valid for whole region outside the black hole, we solve equation (4) directly by transforming it into a hypergeometric equation. With $z=\left(r^{2}-1\right) / r^{2}$, our working region is between $z=0$ and $z=1$, covering the exterior of the NBTZ. Eq.(4) takes a form

$$
z(1-z) f^{\prime \prime}(z)+(1-z) f^{\prime}(z)+\frac{1}{4}\left[\frac{\omega^{2}}{z}-\frac{\ell^{2}}{1-z}-m^{2}\right] f(z)=0 .
$$

In order to obtain quasinormal modes [17], we use the flux boundary condition : the ingoing flux $\left(\mathcal{F}_{\text {in }}(z=0)<0\right)$ at the horizon and the vanishing flux $(\mathcal{F}(z=1)=0)$ at infinity. Then we find two types of quasinormal modes with AdS curvature radius $l$

$$
\omega_{1 / 2}= \pm \frac{\ell}{l}-i \frac{2}{l}\left(n+s_{+}\right)
$$

which means that the operator $\mathcal{L}_{N B T Z}$ is not self-adjoint. Here we have $2 s_{+}=1+(1+$ $\left.m^{2} l^{2}\right)^{1 / 2}$. The discreteness comes from the fact that the NBTZ is a compact (finite) system. Decomposing $\omega_{1 / 2}= \pm \omega_{R}-i \omega_{I}, w_{I}$ should be positive because the corresponding mode decays into the horizon. This bulk perturbation decays, as does in the linear response of conformal field theory 14. The presence of quasinormal modes is a mathematically precise formalism of the lack of unitarity in the semiclassical approach to the bulk system.

To find a unitary system, we study the pure AdS spacetime which does not contain any topologically distinct object. First we transform the wave equation (15) with lower signs into the Schrödinger-like equation [34. We introduce a coordinate $r_{*}=\tan ^{-1}[r](r=$ $\left.\tan \left[r_{*}\right]\right)$ to transform Eq.([5]) into the Schrödinger-like equation ([6]) with the energy $E=\omega^{2}$ and PADS potential

$$
V_{P A D S}\left(r_{*}\right)=\left[\left(\ell^{2}-\frac{1}{4}\right) \cot ^{2}\left[r_{*}\right]+m^{2}+\ell^{2}+\frac{1}{2}+\left(\frac{3}{4}+m^{2}\right) \tan ^{2}\left[r_{*}\right]\right]
$$

`Actually, the radial flux of $\Phi$ is expressed in terms of $f(z)$ as $\left.\mathcal{F}\left(z=z_{0}\right) \equiv 2 \frac{2 \pi}{i}\left[f^{*} z \partial_{z} f-f z \partial_{z} f^{*}\right]\right|_{z=z_{0}}$. As in quantum mechanics, this measures the particle current (flow of energy or information). Hence this quantity is usually used to calculate the black hole greybody factor and quasinormal modes. 
which is defined on a box between $r_{*}=0$ and $r_{*}=\pi / 2$. It is observed that for $m^{2}>0$, $V_{P A D S}\left(r_{*}\right)$ increases to infinity as one approaches $r_{*} \rightarrow 0(r \rightarrow 0)$, while it also goes infinity as one approaches $r_{*} \rightarrow \pi / 2(r \rightarrow \infty)$. $V_{P A D S}\left(r_{*}\right)$ looks like $\cup$. Using the WKB prescription, we expect to have oscillating modes between two turning points for $\omega$ which satisfies $\omega^{2}>V_{P A D S}^{\min }=2\left(m^{2}+\ell^{2}\right)+1$ and $\omega^{2}=V_{P A D S}\left(r_{*}\right)$.

In order to obtain an explicit form for the frequency $\omega$, we solve equation (41) with lower signs directly by transforming it into a hypergeometric equation. With $z=r^{2} /\left(1+r^{2}\right)$, our working region is also between $z=0$ and $z=1$. Eq.(4) takes a form

$$
z(1-z) f^{\prime \prime}(z)+(1-z) f^{\prime}(z)+\frac{1}{4}\left[\omega^{2}-\frac{\ell^{2}}{z}-\frac{m^{2}}{1-z}\right] f(z)=0 .
$$

We require that the wave function be zero at infinity because the potential diverges at infinity. This means that the wave function is normalizable and its flux is zero: $\mathcal{F}(z=$ $1)=0$. In addition, requiring a regular solution at $z=0(r=0)$ lead to real and discrete modes with AdS curvature radius $l$

$$
\omega_{1 / 2}= \pm \frac{\ell}{l} \pm \frac{2}{l}\left(n+s_{+}\right)
$$

which means that $\mathcal{L}_{P A D S}$ is self-adjoint. The discreteness comes from the finite PADS system. These normal modes are consistent with those found in the AdS approach [35. Also this oscillating behavior of a bulk perturbation is mirrored by the oscillating behavior of the CFT-boundary approach [36]. It is obvious that we cannot find any complex mode because there is no the event horizon (dissipative object).

\section{MBTZ}

Although the PADS provides a unitary evolution, it is a topologically trivial spacetime. It is important to find a topologically non-trivial spacetime which provides a unitary evolution. One candidate is the massless BTZ black hole. We start with the wave equation (2) in the background of the massless BTZ black hole spacetime: $d s_{M B T Z}^{2}=-(r / l)^{2} d t^{2}+$ $(l / r)^{2} d r^{2}+r^{2} d \phi^{2}$. Assuming a mode solution (3) with $l=1$, the wave equation for $f(r)$ is given by

$$
r^{2} f^{\prime \prime}(r)+3 r f^{\prime}(r)+\left[\frac{\omega^{2}-\ell^{2}}{r^{2}}-m^{2}\right] f(r)=0 .
$$

Because this equation is so simple, it is not easy to make a potential analysis. Introducing $r_{*}=1 / r^{2}$, Eq.(13) can be rewritten as the Schrödinger-like equation (6) with the zero 
energy $E=0$ and the potential $V_{M B T Z}$

$$
V_{M B T Z}\left(r_{*}\right)=-\frac{k_{1}}{r_{*}}+\frac{k_{2}}{r_{*}^{2}}, k_{1}=\frac{\omega^{2}-\ell^{2}}{4}, k_{2}=\frac{m^{2}}{4} .
$$

It seems that the MBTZ case could not be translated into the boundary value problem because its eigenvalue is determined as $E=0$ initially. Near infinity at $r_{*}=0(r=\infty)$, one finds an approximate equation of $d^{2} f_{0}\left(r_{*}\right) / d r_{*}^{2}-\left(k_{2} / r_{*}^{2}\right) f_{0}\left(r_{*}\right)=0$ whose solution is given by

$$
f_{0}\left(r_{*}\right)=A_{M B T Z} r_{*}^{s_{+}}+B_{M B T Z} r_{*}^{s_{-}}\left(f_{\infty}(r)=A_{M B T Z} r^{-2 s_{+}}+B_{M B T Z} r^{-2 s_{-}}\right)
$$

with $s_{ \pm}=\left(1 \pm \sqrt{1+m^{2}}\right) / 2$. Here the first is a normalizable mode and the second is a nonnormalizable mode. However, it is not easy to obtain approximate solution near the event horizon at $r_{*}=\infty(r=0)$ since the potential $V_{M B T Z}$ contains a long-range interaction term like $k_{1} / r_{*}$.

Introducing $z=1 / r$, the working region is extended from $z=0$ to $z=\infty$. Eq.(13) leads to

$$
z^{2} f^{\prime \prime}(z)-z f^{\prime}(z)+\left[\left(\omega^{2}-\ell^{2}\right) z^{2}-m^{2}\right] f(z)=0 .
$$

In order to solve this equation, one first transforms it into the Bessel's equation with $f(z)=z \tilde{f}(z)$. Using $\eta=\sqrt{\omega^{2}-\ell^{2}} z$, one finds the Bessel's equation

$$
\eta^{2} \tilde{f}^{\prime \prime}(\eta)+\eta \tilde{f}^{\prime}(\eta)+\left[\eta^{2}-\nu^{2}\right] \tilde{f}(z)=0
$$

with $\nu=\sqrt{1+m^{2}}=2 s_{+}-1$. For massless (dilatonic) scalar, it is given by $\nu=1(3)$. Then we find the waveform which is valid for whole region between $z=0$ and $z=\infty 38$

$$
f(z)=C_{1} z J_{\nu}\left(\sqrt{\omega^{2}-\ell^{2}} z\right)+C_{2} z Y_{\nu}\left(\sqrt{\omega^{2}-\ell^{2}} z\right)
$$

In the limit of $z \rightarrow 0(r \rightarrow \infty)$, one has

$$
f(z) \rightarrow f_{0}(z)=A_{M B T Z} z^{2 s_{+}}+B_{M B T Z} z^{2 s_{-}}=A_{M B T Z} r^{-2 s_{+}}+B_{M B T Z} r^{-2 s_{-}},
$$

where $f_{0}(z)$ is consistent with the approximate solution $f_{\infty}(r)$ in Eq.(15). Hence we choose $B_{M B T Z}=0\left(C_{2}=0\right)$ by imposing the boundary condition at infinity of $r=\infty$.

Near the event horizon at $z=\infty(r=0)$, one has

$$
\begin{aligned}
& f(z) \rightarrow f_{\infty}(z)=C_{1} \sqrt{z} \cos \left(\sqrt{\omega^{2}-\ell^{2}} z-\pi s_{+}\right) \\
& \left.\left.=C_{1} \frac{\sqrt{z}}{2}\left[e^{i\left(\sqrt{\omega^{2}-\ell^{2}} z-\pi s_{+}\right.}\right)+e^{-i\left(\sqrt{\omega^{2}-\ell^{2}} z-\pi s_{+}\right.}\right)\right] \equiv f_{M B T Z}^{\text {in }}+f_{M B T Z}^{\text {out }}
\end{aligned}
$$


where the first term is an ingoing mode and the last is an outgoing mode. In this case we have $f_{M B T Z}^{\text {out }}=\left[f_{M B T Z}^{\text {in }}\right]^{*}$ and thus $f_{\infty}(z)$ is real. Then it means that the total flux near the event horizon is zero. However, there exist an ingoing flux and an outgoing flux such that $\mathcal{F}^{\text {in }}(z=\infty)+\mathcal{F}^{\text {out }}(z=\infty)=0$. Therefore, we cannot obtain the wanted case: $\mathcal{F}^{\text {in }}(z=\infty) \neq 0, \mathcal{F}^{\text {out }}(z=\infty)=0$ because the event horizon is a degenerate point and is located at $r=0$. In other words, there is no room to determine the frequency $\omega$ since the spectrum of $E$ is set to be zero initially. This implies that $\mathcal{L}_{M B T Z}$ is self-adjoint and the MBTZ is unitary during evolution. At this stage we have to distinguish between the eigenvalue $E$ of $\mathcal{L}_{M B T Z}$ and the own frequency $\omega$ for $\Phi$. The continuous frequency reflects that the MBTZ is an infinite (non-compact) system. We conclude that its frequency remains real and continuous 39. In order to study the extremal black hole further, we need to introduce the other extremal BTZ black hole in the next section.

\section{EBTZ}

We study the wave propagation for a massive scalar field in the background of the extremal BTZ black hole given by $d s_{E B T Z}^{2}=-\left[(r / l)^{2}-2\left(r_{+} / l\right)^{2}\right] d t^{2}+\left[r^{2} l^{2} /\left(r^{2}-r_{+}^{2}\right)^{2}\right] d r^{2}-$ $\left[2 r_{+}^{2} / l\right] d t d \phi+r^{2} d \phi^{2}$ [28, 40]. In this case $g^{r r}$ is also degenerate at the event horizon of $r=r_{+}=l \sqrt{M / 2}$. Assuming a mode solution in Eq.(3), the radial equation for $f(r)$ is

$$
\begin{aligned}
& \frac{\left(r^{2}-r_{+}^{2}\right)^{4}}{r^{2} l^{4}} f^{\prime \prime}(r)+\frac{\left(r^{2}-r_{+}^{2}\right)^{3}\left(3 r^{2}+r_{+}^{2}\right)}{r^{3} l^{4}} f^{\prime}(r) \\
& +\left[\frac{1}{l^{2}}(\omega l+\ell)\left((\omega l-\ell) r^{2}+2 \ell r_{+}^{2}\right)-\frac{m^{2}}{l^{2}}\left(r^{2}-r_{+}^{2}\right)^{2}\right] f(r)=0 .
\end{aligned}
$$

Choosing a good coordinate $z=r_{+}^{2} /\left(r^{2}-r_{+}^{2}\right)$, the above equation reduces to the Schrödingerlike equation (66) [41, 42]. Here the potential $V_{E B T Z}$ and its energy $E=k_{0}^{2}$ are given by

$$
V_{E B T Z}(z)=-\frac{k_{1}}{z}+\frac{k_{2}}{z^{2}}, k_{0}^{2}=\Omega_{+}^{2}, k_{1}=\Omega_{+} \Omega_{-}, k_{2}=\frac{m^{2} l^{2}}{4}
$$

with $\Omega_{ \pm}=(\omega l \pm \ell) / \sqrt{2 M}$. We comment that the Schrödinger-like equation for the MBTZ can be obtained from the EBTZ-equation by substituting $z$ and $E=k_{0}^{2}$ into $r_{*}$ and $E=0$. Thus we include the previous MBTZ as the special case of the EBTZ with $E=0$.

First we may consider a naively approximate equation of $d^{2} f_{\infty}(z) / d z^{2}+k_{0}^{2} f_{\infty}(z)=0$ near the horizon at $z \rightarrow \infty\left(r=r_{+}\right)$whose solution is given by a plane wave

$$
f_{\infty}=C_{E B T Z} e^{i \Omega_{+} z}+D_{E B T Z} e^{-i \Omega_{+} z}
$$


where the first term corresponds to an ingoing mode and the last is an outgoing one. On the other hand, near infinity at $z \rightarrow 0(r=\infty)$ one obtains an approximate equation $d^{2} f_{0}(z) / d z^{2}-\left(k_{2} / z^{2}\right) f_{0}(z)=0$ which gives us a solution of $f_{0}(z)=A_{E B T Z} z^{s_{+}}+$ $B_{E B T Z} z^{s_{-}}$. Here the first term is a normalizable mode and the second is a nonnormalizable mode.

Up to now we obtain approximate solutions near $z=\infty, 0$. However, we don't know whether these are true solutions because of the long-range potential $V_{E B T Z}$. In order to obtain the solution which is valid for whole region outside the EBTZ, we have to solve equation (22) explicitly. Plugging $f(z)=f_{\infty}(z) f_{0}(z) \tilde{f}(z)$ with $D_{E B T Z}=B_{E B T Z}=0$ into Eq.(22), it takes the form with $\xi=-2 i \Omega_{+} z$

$$
\xi \tilde{f}^{\prime \prime}(\xi)+\left(2 s_{+}-\xi\right) \tilde{f}^{\prime}(\xi)-\left(s_{+}-\frac{i \Omega_{-}}{2}\right) \tilde{f}(\xi)=0 .
$$

This corresponds to the confluent hypergeometric equation and its solution is given by

$$
\tilde{f}(z)=F\left[s_{+}-\frac{i \Omega_{-}}{2}, 2 s_{+} ;-2 i \Omega_{+} z\right]
$$

Considering the Kummer's transformation of $F[a, c ; \xi]=e^{\xi} F[c-a, c ;-\xi]$ with $a=s_{+}-\frac{i \Omega_{-}}{2}$ and $c=2 s_{+}$, it is easy to show that the mode solution $f(z)$ is real: $[f(z)]^{*}=f(z)$.

We choose an ingoing mode near $z=\infty$ and a normalizable solution at $z=0$ as the solution which is valid for whole region outside the horizon

$$
f(z) \sim e^{i \Omega_{+} z} z^{s_{+}} F\left[a, c ;-2 i \Omega_{+} z\right] .
$$

First we calculate the flux at $z=0(r=\infty)$. In this case it confirms that $f_{0}(z) \sim z^{s+}$ is a real function because $F\left[a, c ;-2 i \Omega_{+} z\right] \rightarrow 1, e^{i \Omega_{+} z} \rightarrow 1$ as $z \rightarrow 0$. Then it is obvious that the corresponding flux disappears as

$$
\mathcal{F}(z=0)=\left.2 \frac{2 \pi}{i}\left[f^{*} z \partial_{z} f-f z \partial_{z} f^{*}\right]\right|_{z=0}=0 .
$$

In order to obtain the flux near the event horizon at $z=\infty\left(r=r_{+}\right)$, we use simply the reality condition of $[f(z)]^{*}=f(z)$. Also we find

$$
\mathcal{F}(z=\infty)=0
$$

It is very curious from Eqs.(24) and(29) that even though an ingoing mode of $f_{\infty} \sim e^{i \Omega_{+} z}$ is present near the horizon, its flux is zero. This implies that we need a further investigation on the wave propagation in the EBTZ background. Also it suggests that there is no restriction on the frequency of $\omega$. Thus its mode is real and continuous. 
Now let us derive an explicit waveform near the event horizon at $z=\infty\left(r=r_{+}\right)$. For this purpose we introduce the asymptotic expansion of the confluent hypergeometric function for purely imaginary argument $\xi=-2 i \Omega_{+}$and large $|\xi|[33$.

$$
F[a, c ; \xi] \rightarrow \frac{\Gamma(c)}{\Gamma(c-a)}|\xi|^{-a} e^{ \pm i \pi a / 2}+\frac{\Gamma(c)}{\Gamma(a)}|\xi|^{a-c} e^{ \pm i \pi(a-c) / 2}
$$

where the upper sign being taken if $-\pi / 2<\arg (\xi)<3 \pi / 2$ and the lower one if $-3 \pi / 2<$ $\arg (\xi) \leq-\pi / 2$. Using the above formula, we can easily prove that the Kummer's transformation of $F[a, c ; \xi]=e^{\xi} F[c-a, c ;-\xi]$ is also valid for large $|\xi|$. The approximate wave function is given by

$$
\begin{aligned}
& f(z)=e^{i \Omega_{+} z} z^{s_{+}} F\left[a, c ;-2 i \Omega_{+} z\right] \rightarrow \\
& f_{\infty}(z)=\frac{\Gamma\left(2 s_{+}\right)}{\Gamma\left(s_{+}+i \Omega_{-} / 2\right)}\left(2 \Omega_{+}\right)^{-s_{+}} e^{-\frac{\pi \Omega_{-}}{4}} e^{i\left[\Omega_{+} z+\frac{\Omega_{-}}{2} \ln \left|2 \Omega_{+} z\right|-\frac{\pi s_{+}}{2}\right]} \\
& +\frac{\Gamma\left(2 s_{+}\right)}{\Gamma\left(s_{+}-i \Omega_{-} / 2\right)}\left(2 \Omega_{+}\right)^{-s_{+}} e^{-\frac{\pi \Omega_{-}}{4}} e^{-i\left[\Omega_{+} z+\frac{\Omega_{-}}{2} \ln \left|2 \Omega_{+} z\right|-\frac{\pi s_{+}}{2}\right]} \equiv f_{E B T Z}^{\text {in }}+f_{E B T Z}^{\text {out }} .
\end{aligned}
$$

Comparing the above with Eq.(24) leads to the fact that the first term corresponds the ingoing mode and the last one is the outgoing mode. We observe here that the presence of $k_{1}$-term in Eq.(23) (like Coulomb potential) prevents the ingoing waveform a plane wave in Eq.(24) [43. Also we note that the contribution from $k_{1}$-term to the phases is a logarithmic function of $z$. Even starting with an ugly form of $f_{0}(z) \sim z^{s_{+}}$, a nearly travelling waveform near the event horizon is developed after transformation. Further it is important to confirm that the wave function is real $\left(f_{\infty}(z)=\left[f_{\infty}(z)\right]^{*}\right)$ near the event horizon because of $f_{E B T Z}^{o u t}=\left[f_{E B T Z}^{\text {in }}\right]^{*}$.

In order to obtain quasinormal modes, it requires that the wave function be a purely ingoing mode near the event horizon and $f(z=0)=0$ at infinity. Here we obtain a condition of $s_{+}-i \Omega_{-} / 2=-n, n \in \mathbf{N}$ from $f_{E B T Z}^{\text {out }}=0$. Then we may find the complex and discrete modes with the AdS curvature radius $l$ and $M=1$ as

$$
\omega=-\frac{\ell}{l}-i \frac{2 \sqrt{2}}{l}\left(n+s_{+}\right) .
$$

At the first glance there may exist quasinormal modes for a massive scalar propagation on the EBTZ background. However, this condition leads in turn to the zero ingoing flux because the flux expression

$$
\mathcal{F}_{i n}(z=\infty) \propto \frac{\Gamma\left(2 s_{+}\right)}{\Gamma\left(s_{+}+i \Omega_{-} / 2\right)} \frac{\Gamma\left(2 s_{+}\right)}{\Gamma\left(s_{+}-i \Omega_{-} / 2\right)}
$$


leads to zero exactly when choosing $s_{+}-i \Omega_{-} / 2=-n$. This implies that there is no room to accommodate quasinormal modes of a massive scalar in the background of the EBTZ. Therefore, we show that there is no restriction on the frequency $\omega$ and thus it remains real and continuous. This is an enhanced situation when comparing it with the MBTZ case. A complete analysis is possible for the EBTZ, because the size of its event horizon is finite and it is located at $r_{+} \neq 0$ even it corresponds to a degenerate event horizon. The absence of quasinormal modes in the EBTZ is consistent with the picture of a stable event horizon with thermodynamic properties $T_{H}=C_{J}=0, S_{B H}=4 \pi r_{+}$. This is so because the presence of quasinormal modes implies that a massive scalar wave is losing its energy continuously into the extremal event horizon. Here we mention that the absence of quasinormal modes for the EBTZ is very similar to the case of the de Sitter cosmological horizon 44, 26].

The Schrödinger operator $\mathcal{L}_{E B T Z}$ is self-adjoint because its spectrum is real and continuous. Its continuous spectrum reflects the fact that the EBTZ is an infinite system. Consequently, the EBTZ is unitary during evolution without loss of information.

\section{Summary}

We study the wave equation for a massive scalar in three-dimensional AdS-black hole spacetimes to understand the unitarity issues in a semiclassical way. Here we introduce four interesting spacetimes: the non-rotating BTZ black hole (NBTZ), pure AdS spacetime (PADS), massless BTZ black hole (MBTZ), and extremal BTZ black hole (EBTZ). In the NBTZ case, one finds quasinormal modes, whereas one finds real and discrete modes for the PADS case. The presence of quasinormal modes means that it shows a leakage of information into the event horizon (dissipative object) and thus it signals a breakdown of the unitarity. We can easily achieve the unitarity for the PADS. This is not a dissipative system because the perturbations never disappears completely and always can be restored within the Poincaré recurrence time $t_{P}$ as in the motion of oscillation.

On the other hand, we find real and continuous modes for the MBTZ and EBTZ cases. These are unitary systems. The reasons are as follows. Firstly, the Schrödinger operator becomes self-adjoint upon imposing the Dirichlet condition at infinity, as the same condition at the origin of radial coordinate in the Coulomb scattering in quantum mechanics. Secondly, the corresponding wave functions are real in whole region outside the event horizon, especially for near the event horizon and infinity. This means that there is no leakage of information into the two boundaries: event horizon and infinity. This confirms from the fact that their frequencies are real. Thirdly, we find that the 
radial flux is identically zero outside the event horizon, even though their wave functions are non-zero. Actually, we obtain the ingoing flux as well as the outgoing flux, but summing over these gives us the zero flux near the event horizon exactly. This means that there is no leakage of information into the event horizon. Hence we argue that the two extremal BTZ black holes are unitary systems. In this case we cannot obtain discrete spectra because the two belong to the non-compact system.

Consequently, we propose two additional systems MBTZ and EBTZ for the unitarity system.

A recent work of Hawking does not explain where the semiclassical analysis of the black hole breaks down[11. In the Euclidean path integral approach, the contribution from the topologically trivial sector (pure AdS space), which he had previously neglected, is sufficient to restore the unitarity. However, his arguments are schematic and thus requires more detailed computations. This proposal seems to be incorrect even in the (1+2)-dimensional AdS spacetimes [12, 13]. In the higher-dimensional AdS spacetimes,

there exists the Hawking-Page transition between the AdS black hole and pure AdS space. This is a first-order phase transition. This supports partly that Hawking's arguments is correct. In the (1+2)-dimensional AdS spacetimes, there exists a second-order phase transition between NBTZ and MBTZ (not PADS) [45]. This may explain why we use the MBTZ instead of the PADS, even both are unitary systems. At this stage, however, we don't know how the EBTZ plays a role in resolving the non-unitarity issue of the non-extremal black hole, the NBTZ.

\section{Acknowledgement}

Y. Myung was supported by the Korea Research Foundation Grant (KRF-2005-013C00018). H. Lee was in part supported by KOSEF, Astrophysical Research Center for the Structure and Evolution of the Cosmos.

\section{References}

[1] S. W. Hawking, Phys. Rev. D 14, 2460 (1976).

[2] G. 't Hooft, Nucl. Phys. B 335, 138 (1990).

[3] L. Susskind, hep-th/0204027.

[4] D. N. Page, hep-th/0409024. 
[5] J. Maldacena, JHEP 0304, 021, (2003) 021 hep-th/0106112.

[6] M. Banados, M. Henneaux, C.Teitelboim and J. Zanelli, Phys. Rev. D 48, $1506(1993)$ gr-qc/9302012.

[7] S. Carlip, gr-qc/0503022.

[8] J. Maldacena, Adv. Theor. Math. Phys. 2, 231 (1998) [Int. J. Theor. Phys. 38, 1113 (1999)] hep-th/9711200.

[9] S. S. Gubser, I. R. Kelebanov and A. M. Polyakov, Phys. Lett. B428, 105 (1998) hep-th/9802109.

[10] E. Witten, Adv. Theor. Math. Phys. 2, 253 (1998) hep-th/9802150.

[11] S. W. Hawking, hep-th/0507171.

[12] M. Kleban, M. Porrati and R. Rabadan, JHEP 0410, 030(2004) hep-th/0407192.

[13] S. Solodukhin, Phys. Rev. D 71, 064006 (2005) hep-th/0501053.

[14] D. Birmingham, I. Sachs, and S. Solodukhin, Phys. Rev. Lett. 88, 151301 (2002) hep-th/0112055.

[15] S. Solodukhin, hep-th/0406130.

[16] G. Siopsis, Class. Quant. Grav. 22, 1425 (2005) hep-th/0408091.

[17] D. Birmingham, I. Sachs, and S. Sen, Phys. Lett. B413, 281 (1997) hep-th/9707188.

[18] G. T. Horowitz and D. L. Welch, Phys. Rev. Lett. 71, 328(1993) hep-th/9302126.

[19] J. H. Horne and G.T. Horowitz, Nucl. Phys. B 368, 444(1992) hep-th/9108001.

[20] H. W. Lee and Y.S. Myung, Phys. Rev. D 58, 104013(1998) hep-th/9804095.

[21] H. W. Lee, N. J. Kim, and Y. S. Myung, Phys. Rev. D58, 084022(1998) hep-th/9803080.

[22] H. W. Lee, N. J. Kim, and Y. S. Myung, Phys. Lett. B441, 83(1998) hep-th/9803227.

[23] J. B. Arfken and H. J. Weber, Mathematical Methods for Physics (Academic Press, San Diago, 1995), chap.9. 
[24] J. S. F. Chan and R. B. Mann, Phys. Rev. D55, 7546(1997) gr-qc/9612026.

[25] G. T. Horowitz and V. Hubeny, Phys. Rev. D62, 024027 (2000) hep-th/9909056.

[26] Y. S. Myung and N. J. Kim, Class. Quant. Grav. 21, 63(2004) hep-th/0304231.

[27] R. G. Cai, Z.-J. Lu, Y.-Z. Zhang, Phys. Rev. D 55, 853(1997) gr-qc/9702032.

[28] R. -G. Cai and J.-H. Cho, Phys. Rev. D 60, 067502(1999) hep-th/9803261.

[29] Y. S. Myung, Phys. Lett. B 579, 205(2004) hep-th/0310176.

[30] J. Maldacena and A. Strominger, JHEP 9812, 005(1998) hep-th/9804085.

[31] V. Cardoso and J. P. Lemos, Phys. Rev. D63, 124015 (2001) gr-qc/0101052.

[32] N. D. Birrell and P. C. W. Davies, Quantun fields in curved space (Cambridge Univ., New York, 1982).

[33] M. Abramowitz and I. Stegun, Handbook of Mathematical Functions (Dover Publication, New York, 1970)

[34] J. Barbon and E. Rabinovici, Fortsch. Phys. 52, 642(2004) hep-th/0403268; hep-th/0503144.

[35] V. Balasubramanian, P. Kraus and A. Lawrence, Phys.Rev. D59, 046003 (1999) hep-th/9805171.

[36] D. Birmingham, I. Sachs, and S. N. Solodukhin, Phys. Rev. D67, 104026(2003) hep-th/0212308.

[37] G. Lifschytz and M. Ortiz, Phys. Rev. D 49, 1929 (1994) gr-qc/9310008.

[38] H. W. Lee and Y. S. Myung, hep-th/9808002.

[39] J. Barbon and E. Rabinovici, JHEP 0311, 047(2003) hep-th/0308063;

[40] E. Keski-Vakkuri, Phys. Rev. D 59, 104001(1999) hep-th/9808037.

[41] J. Gamboa and F. Mendez, Class. Quant. Grav. 18, 225(2001) hep-th/0006020.

[42] J. Crisostomo, S. Lepe, and J. Saavedra, Class. Quant. Grav. 21, 2801(2004) hep-th/0402048. 
[43] E. Merzbacher, Quantum mechanics (John Wiley and Sons, New York, 1961), p.248.

[44] R. Bousso, A. Maloney, and A. Strominger, Phys. Rev. D65, 104039 (2002) hep-th/0112218.

[45] Y. S. Myung, Phys. Lett. B 624, 297 (2005) hep-th/0506096. 